\title{
Prevalence and Susceptibility Profiles of Non-Fermentative Gram-Negative Bacilli Infection in Tertiary Care Hospital
}

\author{
Siddiqui Heena Kausar*, V.P. Bansal and M. Bhalchandra \\ Department of Microbiology, MGM Hospital and college, Aurangabad, India \\ *Corresponding author
}

\section{Keywords \\ Non-fermenting gram-negative bacilli, Prevalence, Pseudomonas aeruginosa, Acinetobacter species, Nosocomial infection.}

\section{Article Info}

Accepted:

06 December 2017

Available Online:

10 January 2018

\section{A B S T R A C T}

Non-fermenting gram negative bacilli (NFGNB) have emerged as a major cause of nosocomial infection. They can be recovered from the hospital environment, commonly cause device related infections, are often resistant to disinfectant. These NFGNB are becoming increasingly resistant to antibiotics which are used in critically ill patients. Aims: To know the prevalence and antimicrobial susceptibility profile of NFGNB from various clinical specimens. The main objectives of this study is to know the various NFGNB and the prevalence in hospital. And to study the antimicrobial susceptibility test (AST) pattern of NFGNB the isolates identified by standard bacteriological methods Vitek-2 system. AST pattern was generated by using Vitek-2 system except Burkholderia cepaciae complex (BCC). BCC AST was done by standard disc diffusion method. A total of 121 NFGNB for 1850 clinical specimens were isolated and identified by its bacteriological methods and Vitek-2 system. AST was performed by Vitek-2 system because method for the AST pattern of their isolated revealed variation to this sensitivity pattern. Thus correct identification of these isolates is of utmost importance for the correct antibiotic treatment. The organisms were identified by standard bacteriological and Vitek-2 system. Out of 1850 clinical specimens NFGNB we found 121 specimens with a prevalence rate. The various isolated in the present study were Pseudomonas species-79, Acinetobacter-27, Burkholderia species-7, Stenotrophomonas maltophilia ( $S$. maltophilia)-4, Sphingomonas species-4. The AST pattern of these isolates is being studied.

\section{Introduction}

Non-fermenting gram-negative bacilli (NFGNB) including Pseudomonas species, Acinetobacter species, Burkholderia species, Stenotrophomonas maltophilia species, are emerging as an important causes of blood stream infections (BSI) world-wide particularly immune compromised patients with haematological malignancies and patients admitted in intensive care unit (ICUs) (Ramphal et al., 2008). The organisms are ubiquitous in nature particularly in soil and water in the hospital environment, they may 
be isolated from instruments such as ventilator machine, humidifiers, mattresses and other equipment as well as from the skin of health care workers (Kiran Chawla et al., 2013). Non-fermenting bacteria associated with nosocomial infections are becoming increasingly resistant to commonly used antimicrobial agents (Kalidas Rit et al., 2013). The high intrinsic resistance of these bacteria to different antimicrobial agents, thus there is a value of proper identification that comes to the forefront. Burkholderia cepacia complex (BCC) is intrinsically resistant to many $\beta$ lactam drugs, aminoglycosides, colistin and polymyxin $\mathrm{B}$, the first line therapeutics of choice against Pseudomonal infections. Limited sensitivity of BCC isolates to meropenem is significant as it is one of the first line drugs against Pseudomonas species. There are few studies that provide the antibiogram data of NFGNB especially, and this may vary in every hospital set up knowing the antibiogram data of NFGNB in our set up will be very useful in deciding appropriate treatment strategies for these bacteria. With above facts in mind we undertook this study to identify various NFGNB from various clinical specimens in our hospital settings and to know their antibiotic sensitivity pattern in our set up.

\section{Materials and Methods}

\section{Study material and place}

121 isolates of NFGNB from 1850 clinical specimens received in microbiology department of MGM hospital Aurangabad were included in this study. The specimens were blood, pus, urine, BAL, ET secretion and various body fluids. The study was carried out after the approval of Ethical committee of institution.

\section{Study period}

May 2015- December 2015.

\section{Materials and Methods}

All specimens except blood were inoculated on Blood Agar and MacConkey's Agar.

Blood specimens were inoculated in Bac $\mathrm{T}$ Alert blood culture system. Subcultures from these were made on Blood Agar and MacConkey's Agar when the system fledged the bottles positive.

Presumptive identification of isolates were made by a combination of conventional bacteriological methods like colony morphology, gram staining, biochemical tests and Vitek-2 system was used for confirmation and identification of these bacteria was important. Organisms that failed to acidify the butt of TSI media were considered as nonfermenting organisms.

AST was done of these isolates excluding Burkholderia species on the Vitek-2 system.

Antibiotic tested usingVitek-2 system are Ticarcillin/Clavulanic acid (TI), Piperacillin/ Tazobactam (PI/TAZ), Ceftazidime (CAZ), Cefoperazone/ Sulbactam (CFS), Cefepime (CPM), Doripenem (DOR), Imipenem (IM), Meropenem (MRP), Amikacin(AK), Gentamycin (GEN), Ciprofloxacin (CIP), Levofloxacin (LE), Minocyclin (MI), Tigecycline (TG), Colistin (CL) and Trimethoprim/ Sulfamethoxzole (COT).

The Vitek system for some unknown reason was not giving AST result of Burkholderia species so for Burkholderia species AST was done manually on Muller Hilton Agar (MHA) using Kirby-Baeur disc diffusion method, using for the following antibiotics as per CLSI guidelines. Ceftazidime (CAZ), Trimethorpim/Sulfamethoxzole (COT), Levofloxacin (LE), Minocyclin (MI), Chloramphenicol (C) and Meropenem (MRP). 


\section{Results and Discussion}

Results will be discussed in tabular form under following heading.

To study the antimicrobial susceptibility test (AST) pattern of NFGNB as to prepare guidelines for treatment of infection caused by these organisms.

Organisms isolated from specimens.

AST pattern of the organisms isolated from specimens.

A total of $121(11.62 \%)$ NFGNB were isolated from 1850 clinical specimens. The various clinical specimens from which NFGNB were isolated are pus (38), urine (21), blood (16), sputum (6), BAL (6), ET secretions (8) and other body fluids (26). The predominant isolate was Pseudomonas species (79), followed by Acinetobacter (27), Burkholderia cepacia complex (7), Stenotrophomonas maltophilia (4), and Sphingomonas paucimobilis (4) (Table 1).
In this study the two major isolates Pseudomonas (79) and Acinetobacter (27) accounted for $87.6 \%$ of all isolated NFGNB. Both showed $100 \%$ sensitivity towards colistin. For other antibiotics Pseudomonas gave a decent sensitivity for Meropenem (75.9\%), Amikacin (75.9\%), Doripenem (74.6\%). Whereas Acinetobacter had a good sensitivity of $92.5 \%$ for Tigecyclin. For Burkholderia cepacia complex 100\% sensitivity was for Trimethoprim/ Sulfamethoxzole and a sensitivity around $85 \%$ for Ceftazidime and Chloramphenicol. It gave a decent sensitivity of $71.4 \%$ for Meropenem.

For Stenotrophomonas maltophilia the $100 \%$ sensitivity was for colistin, cefipime and ciprofloxacin. It had a decent sesnsitivity of 75\% for Cefaperazone/Sulbactam, Levofloxacin and co-trimaxzole. For Sphingomonas paucimobilis $100 \%$ sensitivity was for Amikacin, Cefaperazone/Sulbactam, Minocyclin, Tigecyclin and Trimethoprim/ Sulfamethaxazole and $75 \%$ sensitivity for Ceftazidime, Gentamycin and ticarcillin (Table 2).

Table.1 Sample source of NFGNB

\begin{tabular}{|l|l|l|l|l|l|l|l|l|}
\hline Organism & Pus & Blood & Urine & Sputum & BAL & $\begin{array}{l}\text { ET } \\
\text { Secretions }\end{array}$ & $\begin{array}{l}\text { Body } \\
\text { Fluids }\end{array}$ & Total \\
\hline $\begin{array}{l}\text { Pseudomonas } \\
\text { species }\end{array}$ & 29 & 7 & 12 & 4 & 5 & 5 & 17 & 79 \\
\hline $\begin{array}{l}\text { Acinetobacter } \\
\text { species }\end{array}$ & 5 & 6 & 4 & 1 & 1 & 2 & 8 & 27 \\
\hline $\begin{array}{l}\text { Burkholderia } \\
\text { species }\end{array}$ & 2 & 1 & 1 & 1 & - & 1 & 1 & 7 \\
\hline $\begin{array}{l}\text { St.maltophilia } \\
\text { species }\end{array}$ & 1 & 2 & 1 & - & - & - & - & 4 \\
\hline $\begin{array}{l}\text { S.paucimobilis } \\
\text { species }\end{array}$ & 1 & - & 3 & - & - & - & - & 4 \\
\hline \begin{tabular}{l} 
total \\
\hline
\end{tabular} & 38 & 16 & 21 & 6 & 6 & 8 & 26 & 121 \\
\hline
\end{tabular}


Table.2 Antibiotic susceptibility pattern for various isolates

\begin{tabular}{|c|c|c|c|c|c|c|}
\hline Sr.no & Antibiotics & & & Pattern of & acteria & \\
\hline & & $\begin{array}{l}\text { Pseudomonas } \\
(\mathrm{n}=7)\end{array}$ & $\begin{array}{l}\text { Acinetobacter } \\
(\mathrm{n}=27)\end{array}$ & $\begin{array}{l}\text { BCC } \\
(n=7)\end{array}$ & $\begin{array}{l}\text { S. maltophilia } \\
(\mathrm{n}=4)\end{array}$ & $\begin{array}{l}\text { S. paucimobilis } \\
(\mathrm{n}=4)\end{array}$ \\
\hline 1. & Colistin & $79(100 \%)$ & $27(100 \%)$ & - & $4(100 \%)$ & $2(50 \%)$ \\
\hline 2. & Meropenem & $60(75.9 \%)$ & $10(37.0 \%)$ & $5(71.4 \%)$ & $1(25 \%)$ & $3(75 \%)$ \\
\hline 3. & Amikacin & $60(75.9 \%)$ & $79(25.9 \%)$ & - & $1(25 \%)$ & $4(100 \%)$ \\
\hline 4. & Doripenem & $59(74.6 \%)$ & $9(33.3 \%)$ & - & $1(25 \%)$ & $2(50 \%)$ \\
\hline 5. & $\begin{array}{l}\text { Cefaperazone/ } \\
\text { Sulbactam }\end{array}$ & $56(70.8 \%)$ & $9(33.3 \%)$ & - & $3(75 \%)$ & $4(100 \%)$ \\
\hline 6. & Cefipime & $55(69.6 \%)$ & $9(33.3 \%)$ & - & $4(100 \%)$ & $2(50 \%)$ \\
\hline 7. & Ceftazidime & $52(65.8 \%)$ & $8(29.6 \%)$ & $6(85.7 \%)$ & $3(75 \%)$ & $3(75 \%)$ \\
\hline 8. & $\begin{array}{l}\text { Peperacillin/ } \\
\text { Tazobactam }\end{array}$ & $51(64.5 \%)$ & $8(29.6 \%)$ & - & $1(25 \%)$ & $2(50 \%)$ \\
\hline 9. & Gentamycin & $51(64.5 \%)$ & $11(40.7 \%)$ & - & $1(25 \%)$ & $3(75 \%)$ \\
\hline 10. & Imipenem & $50(63.2 \%)$ & $10(37.0 \%)$ & - & $1(25 \%)$ & $2(50 \%)$ \\
\hline 11. & Ciprofloxacin & $45(56.9 \%)$ & $9(33.3 \%)$ & - & $4(100 \%)$ & $2(50 \%)$ \\
\hline 12. & Levofloxacin & $45(56.9 \%)$ & $7(25.9 \%)$ & $4(57.1 \%)$ & $3(75 \%)$ & $2(50 \%)$ \\
\hline 13. & Ticarcillin & $44(55.6 \%)$ & $8(29.6 \%)$ & - & $2(50 \%)$ & $3(75 \%)$ \\
\hline 14. & Minocyclin & $21(26.5 \%)$ & $18(66.67 \%)$ & $4(57.1 \%)$ & - & $4(100 \%)$ \\
\hline 15. & Tigecyclin & $04(5.6 \%)$ & $25(92.5 \%)$ & - & $3(75 \%)$ & $4(100 \%)$ \\
\hline 16. & $\mathrm{COT}$ & $03(3.79 \%)$ & $9(33.3 \%)$ & $7(100 \%)$ & $3(75 \%)$ & $4(100 \%)$ \\
\hline 17. & Chloramphenicol & - & - & $6(85 \%)$ & - & - \\
\hline
\end{tabular}

In recent years isolation of NFGNB has gained importance with increasing reports of these bacteria relating them to hospital outbreaks or health care associated infections (Malini et al., 2009). The most common of these NFGNB are Pseudomonas and Acinetobacter (Ramphal et al., 2008). So is also true in the present study. In the present study majority of isolates were from pus specimen which is similar to the study done by Kalidas Rit et al., (2013) in the present study $100 \%$ sensitivity was shown by Pseudomonas and Acinetobacter which are similar to the findings of Kalidas Rit et al., (2013) who has reported a $100 \%$ sensitivity for Pseudomonas and $94 \%$ for Acinetobacter. Buekholderia cepacia complex in the present study had the highest sensitivity for Trimethoprim Sulphamethaxazole. These findings are in accordance to the study by Fehlberg et al.,

(2016) who have mentioned a high sensitivity of $97.6 \%$ of this antibiotic. Similar a sensitivity of $85 \%$ for Ceftazidime matches the sensitivity of $93.9 \%$ in the study of Fehlberg et al., (2016). But Fehlberg et al., (2016) had reported a low sensitivity of $30.5 \%$ to Chloramphenicol as compared to $85 \%$ in our study. In our study a lower sensitivity of $71.4 \%$ was achieved for Meropenem which matches the study of Loren et al., but higher a sensitivity of $100 \%$ was reported by Kiran et al., (2013).

Stenotrophomonas maltophilia had $100 \%$ sensitivity for Colistin, Cefipime, Ciprofloxacin. Similar higher sensitivity to aminoglycosides was reported by Malini et al., (2009) (100\%) and Kiran et al., (2013) (93.3\%). A sensitivity of $75 \%$ to Trimethoprime/ sulphamethaxazole in this study is less as compared a sensitivity of $100 \%$ reported by Malini et al., (2009), Kiran et al., (2013) has reported a sensitivity of $86.7 \%$ for trimethoprime/ sulphamethaxazole, colistin was $100 \%$ sensitive in the present study which is much higher than $56.1 \%$ 
reported by Nicodemo et al., (2004). As for as sensitivity of Sphingomonas paucimobilis $100 \%$ to trimethoprime/ sulphamethaxazole, tigecycline, minocycline and meropenem and cefoperazone/ sulbactam is concerned it is difficult to comment on there therapeutic usefulness as studies for Sphingomonas paucimobilis could not be found. It will require more studies in future to assist the clinical response of these drugs to infections by this particular bacteria. From their findings it is evident that variable antibiotic sensitivity pattern of the bacteria and intrinsic resistance of some of these to various antibiotics is making proper identification and antibiotic sensitivity for these isolates is important in reducing the mortality and morbidity because of these infections. The antibiotic sensitivity pattern will vary among hospitals and geographic areas, so findings and establishing antibiotics policies for these NFGNB in setups will go a long way with infections because of these bacteria.

NFGNB are nowadays important pathogens causing life threatening hospital associated infections. These bacteria differ in antimicrobial treatment as bacteria are intrinsically resistant to a variety of antibiotics. This brings into focus the importance of their correct identification and antibiotic sensitivity testing. For this automated ID and AST systems like Vitek-2 could be handful addition in conventional bacteriological methods. One should go forward and find the in vivo response of these antibiotics so as to decide the best treatment options in their own hospital set ups.

\section{Acknowledgements}

Authors are thankful to Dean, M.G.M Medical College, Aurangabad for granting the permission for this work.

\section{How to cite this article:}

Siddiqui Heena Kausar, V.P. Bansal and Bhalchandra, M. 2018. Prevalence and Susceptibility Profiles of Non-Fermentative Gram-Negative Bacilli Infection in Tertiary Care Hospital. Int.J.Curr.Microbiol.App.Sci. 7(01): 740-744. doi: https://doi.org/10.20546/ijcmas.2018.701.089

\section{References}

Fehlberg, L.C., Nicoletti, A.G., Ramos, A.C., Rodrigues-Costa, F., de Matos, A.P., Girardello, R., Marques, E.A., Gales, A.C. In vitro susceptibility of Burkholderia cepacia complex isolates: Comparison of disk diffusion, Etest ${ }^{\circledR}$, agar dilution, and broth microdilution methods. Diagn Microbiol Infect Dis. 2016 Dec; 86(4): 422-427. doi: 10.1016/j.diagmicrobio. 2016.08.015.

AC Nicodemo, M.R.E. Arajuo, A.S. Ruiz, A.C Gates. Jrof. In vitro susceptibility of Stenotrophomonas maltophilia isolates: comparison of this diffusion, $\mathrm{E}$ test and Agar dilution methods. Antimicrobial chemotherapy. Vol 53, issue 4, 2004, 604-608.

Malini A, Deepa E, Gokul B, Prasad S. Nonfermenting gram-negative bacilli infections in tertiary care hospital in Kolar, Karanataka. J Lab Physicians. 2009; 1:62-6.

Kiran Chawla, Shashidhar Vishwanath and Frenil C Munim. Non-fermenting gramnegative bacilli other than Pseudomonas aeruginosa and Acinetobacter species. Casuing respiratory tract infections in a tertiary care centre. J Glob Infect Dis. 2013 Oct-Dec; 5(4): 144-148.

Kalidas Rit, Falguni Nag, PK Maity. 2013. Prevalence and susceptibility profiles of non-fermentative gram-negative bacilli infection in tertiary care hospital of Eastern India.

Braunwald E, Hauser SL, Longo DL, Jameson $\mathrm{JL}$, et al., Ramphal R. Infections due to Pseudomonas species and related organisms. Chapter 145 Harrison's Principles of Internal Medicine $17^{\text {th }}$ edn. In: Fauci AS, Editors USA: McGrawHill Medical; 2008. Pp.949-56. 